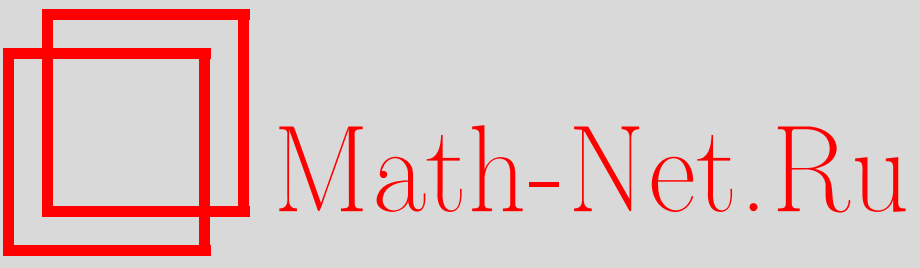

Д. И. Пионтковский, О рядах Гильберта и соотношениях в алгебрах, УМH, 1998, том 53, выпуск 6, 257-258

DOI: https://doi.org/10.4213/rm102

Использование Общероссийского математического портала Math-Net.Ru подразумевает, что вы прочитали и согласны с пользовательским соглашением

http://www.mathnet.ru/rus/agreement

Параметры загрузки:

IP : 54.237 .59 .107

26 апреля 2023 г., 15:16:08 


\title{
О РЯДАХ ГИЛЬБЕРТА И СООТНОШЕНИЯХ В АЛГЕБРАХ
}

\author{
Д. И. Пионтковский
}

Зафиксируем поле $k$. Ниже все $k$-алгебры ассоциативные и градуированные, т.е. конечно $\mathbb{Z}_{+}$-градуированные с одномерной нулевой компонентой; такая алгебра $A=A_{0} \oplus A_{1} \oplus \ldots$ называется стандартной, если она порождена компонентой $A_{1}$. Для такой алгебры $A$ через $A(x)$ обозначим ее ряд Гильберта $\sum_{i \geqslant 0} \operatorname{dim} A_{i} x^{i}$. Неравенства между степенными рядами понимаются покоэффициентно: запись $\sum_{i} a_{i} x^{i} \geqslant \sum_{i} b_{i} x^{i}$ означает, что $a_{i} \geqslant b_{i}$ для всех $i \geqslant 0$.

Пусть $A$ - алгебра, $I \triangleleft A$ - двусторонний идеал, минимальным образом порожденный некоторым множеством однородных элементов $\alpha \subset A$, и $B=A / I$. Пусть $\alpha(x)$ - производящая функция множества $\alpha: \alpha(x)=\sum_{i \geqslant 0} \#\left(\alpha \cap A_{i}\right) \cdot x^{i}$. Эта заметка посвящена исследованию следующего вопроса: какова связь между рядами Гильберта алгебр $A$ и $B$ и рядом $\alpha(x)$ ?

Пусть $C=B * k\langle\alpha\rangle-$ свободное произведение $B$ и свободной алгебры $k\langle\alpha\rangle$. Очевидно, выполняется неравенство рядов Гилшберта

$$
C(x) \geqslant A(x) .
$$

Те множества $\alpha$, для которых (1) обращается в равенство, называются сильно св ободныц.ми [1]: это некоммутативный аналог хорошо известных в некоммутативной алгебре регулярных последовательностей [1], [2].

Вообще говоря, конечно-порожденная алгебра $H$ имеет экспоненциальный рост, и асимптотическое поведение ряда Гилберта $H(x)$ характеризуется әкспонентой роста $p(H)$ : если $r(H)$ - радиус сходимости ряда $H(x)$, то $p(H)=r(H)^{-1}$. Назовем алгебру $A$ әкстремальной, если $r(A / I)>r(A)$ для любого собственного идеала $I \triangleleft A$.

ТЕОрема 1. (i) Экстремальнье алгебры первичны.

(ii) Свободное произведение двух нетривиальных алгебр әкстремально.

(iii) Если алгебра содержит непустое сильно свободное множество, то она экстремальна.

Используя теорему 1 , можно получить оценку на ряд $\alpha(x)$ и усилить приведенную выше характеризацию сильно свободных множеств.

Теорема 2. Пусть $A$-конечно-порожденная алгебра, $B, \alpha$-те же, что и раньше. Тогда имеет место неравенство

$$
B(r(A)) \cdot \alpha(r(A)) \geqslant 1,
$$

причем следующие условия әквивалентны:

(i) это неравенство обращается в равенство;

(ii) $r(A)=r(C)$;

(iii) множество $\alpha \subset A$ сильно свободно.

Пусть теперь $A$ - свободная ассоциативная алгебра ранга $s$ со стандартной градуировкой. Тогда подобного рода оценку дает теорема Голода-Шафаревича: из нее, в частности, следует, что при достаточно малом числе соотношений в каждой степени $B$ бесконечномерна [3]. Как показано в [1, theorem 2.6], равенство в неравенстве Голода-Шафаревича

$$
B(x)(1-s x+\alpha(x)) \geqslant 1
$$

равносильно тому, что множество соотношений $\alpha$ сильно свободно.

Пусть идеал $I$ порожден $t$ элементами степени $l$, т.е. $\alpha=\left\{f_{1}, \ldots, f_{t} \mid \operatorname{deg} f_{i}=l\right\}$. В этой ситуации В. Е. Говоровым даны следующие оценки на число соотношений $t$.

Работа выполнена при частичной поддержке Российского фонда фундаментальных исследований (грант № 96-01-00149). 
ТеОРема 3 [4]. Ряд $B(x)=\sum_{i \geqslant 0} b_{i} x^{i}$ сходится при $x=s^{-1}$. При әтом выполняются неравенства

$$
t \geqslant s^{l} / B\left(s^{-1}\right)
$$

$u$

$$
t \geqslant\left(s b_{n-1}-b_{n}\right) / b_{n-1} \quad \text { при всех допустимьх } n .
$$

Оказьвается, в обоих случаях равенство равносилно равенству в неравенстве Голода-Шафаревича.

Теорема 4. Следующие условия эквивалентны:

(i) Мнохество а сильно свободно.

(ii) Неравенство (2) обращается в равенство.

(iii) Неравенство (3) обращается в равенство при всех допустимых $n$.

Как показал Д. Аник [5], даже при $l=2$ над полем характеристики нул для некоторых $t$ и $s$ проблема распознавания сильно свободных множеств $\alpha$ алгоритмически неразрешима. Таким образом, получаем

СлЕДСтвиЕ 5. Пусть char $k=0$. Для некоторых натуральных чисел $s, t$ не существует алгоритма, который по заданному набору а из $t$ соотношений 2 -й степени от $s$ переменных проверял бы равенство $r(C)=s^{-1}$, где $C$ - алгебра с данными соотношениями, порожденная $s$ әлементами 1-й степени и $t$ әлементами 2-й степени. Кроме того, не существует алгоритма, который проверял бы равенство $B\left(s^{-1}\right)=s^{2} t^{-1}$, где $B$-s-порожденная стандартная алгебра с теми же соотношениями.

Тем самым, над полем нулевой характеристики для конечно определенных алгебр проблемы вычисления асимптотики ряда Гильберта или его значения в точке алгебраически неразрешимы.

\section{СПИСОК ЛИТЕРАТУРЫ}

[1] Anick D. // J. Algebra. 1982. V. 78. P. 120-140. [2] Голод Е. C. // УМН. 1997. Т. 52. № 4. С. 210-202. [3] Голод Е. С., Шафаревич И. Р. // Изв. АН СССР. Сер. матем. 1964. Т. 28. № 2. С. 261-272. [4] Говоров В. Е. // Матем. заметки. 1972. Т. 12. № 2. С. 197-204. [5] Anick D. // Ann. of Math. 1985. V. 122. P. 87-112.

Московский государственньй университет им. М.В. Ломоносова
Принято редколлегией 02.09.1998 